\title{
A Computer Experiment Teaching System Based on OMAP Embedded System
}

\author{
https://doi.org/10.3991/ijet.v13i05.8439 \\ Xiaorong Wang and Cuimei Fan \\ Jiangsu College of Finance and Accounting, Lianyungang, China \\ footloose829@hotmail.com
}

\begin{abstract}
Open Multimedia Application Platform (OMAP) is an embedded processor with high performance and high integration density which is developed to meet the application of mobile multimedia information processing and wireless communication. Duo-core architecture of OMAP can effectively solve the contradiction between performance and power dissipation. OMAP-based embedded system is a special computer system which is based on computer technology, and the soft and hardware can be tailored. At present, college computer experiment teaching has high requirements for multimedia computer technology. In this research, theory construction is conducted on the basis of OMAP embedded system, and a special multimedia playing, and storage method is introduced. The function design starts from teacher terminal and student terminal, and Computer experiment teaching system based on OMAP embedded system is constructed. The practical application effect proves that the teaching system has a good effect and plays an important role in achieving teaching reform objective and improving teaching quality.
\end{abstract}

Keywords-OMAP, computer experiment teaching, multimedia

\section{$1 \quad$ Introduction}

Multimedia computer technology is based on computer medium information processing technology and integrates digit, text, image, voice, video, animation, perceiving and measuring techniques. Besides, it involves computer software and hardware to process the above information and compress data, etc. It is a technology which adopts multiple media types to receive, express, communicate and process information. Multimedia computer technology develops and changes most rapidly in the field of modern information technology, with the most applications. It is the development and competition hotspot of computer technology [1]. The rapid development of multimedia computer technology also offers more choices for multimedia teaching.

OMAP embedded system is a complete computer system including hardware and software, but it is different from general-purpose computer system. It takes application as the center and is based on computer technology. Besides, software and hardware can be tailored. It is applicable to special computer system with strict requirements for function, reliability, cost, volume and power dissipation [2]. The computer 
used by the embedded system is a special microprocessor embedded into the controlled object, but the function is more specialized than that of general-purpose computer. It owns appropriate operating rate, high reliability and low comparative cost especially designed for an aspect that the general-purpose computer cannot own. OMAP (Open Multimedia Application Platform) is an embedded processor with high performance and high integration density which is developed to meet the application of mobile multimedia information processing and wireless communication. It was first developed by Texas Instruments [3].

In recent years, multimedia computer technology has been applied in computer experiment teaching. OMAP embedded system in multimedia computer technology owns multilayer and multi-method display modes because of its own technical advantage, and greatly enriches teaching means of computer experiment teaching. This research is based on such background. In this paper, computer experiment teaching system based on OMAP embedded system was designed according to the design philosophy of common embedded system, in the hope of promoting the innovative development of computer experiment teaching mode and offering the feasible thought for solving low efficiency of college computer teaching.

\section{State of the art}

Computer experiment teaching [4] is a college professional course, with the features of technical property, practical property and applicability. Efficient computer experiment teaching can promote students' practical application ability and play a critical role for students' future development. However, since problems exist in teaching means and teaching contents, systematical research and reform are urgently needed for college computer experiment teaching. Sheng [5] took VB Program Design for example to explore the reform of college computer experiment teaching, in the hope of enhancing students' practical and problem-solving ability through proper adjustment and then fundamentally promoting computer course quality. Liang [6] deeply analyzed the effect of introduction of embedded system on computer system ability training, proposed the ways to improve the quality of embedded system course based on practice and offered the feasible way and thought for college computer course reform.

Some scholars have noticed the possibility of applying OMAP embedded system in computer course teaching and carried out relevant researches. Shi et al. [7] designed and implemented OMAP-based embedded system, including system scheme selection, hardware circuit design and embedded software development platform setup, etc. The scholar also tested the operation effect of drive program and application program in embedded system and laid a good foundation for system improvement and promotion. For the problems of existing video coding and decoding systems such as large power dissipation and high cost, Dai [8] designed the video coding and decoding system based on OMAP platform, adopted dual-core (ARM+DSP) embedded processor, applied hardware accelerator in DSP to conduct complex computation in H.264 algorithm, optimized video coding and decoding algorithm from such aspects as stor- 
age, code compiling and video frame loss and effectively reduced system resource occupancy rate. But in fact, just because of large multimedia data size, large storage capacity or high processing capacity is required to construct modern education server mode. When users demand, one course usually has multiple teaching video versions (the versions of various colleges are different, and even there are audio courses in several languages) for current storage mode. Thus, multiple audio data need to be stored. On the one hand, lots of storage resources need to be occupied [9]; on the other hand, only original dubbing can be listened in multimedia playing, while the original dubbing may be not suitable for all students. So, it is hard to meet individualized learning needs. The specific design was introduced in this paper on the basis of relevant researches so as to provide help for computer course teaching reform.

OMAP-based embedded system owns two innovations: on the one hand, OMAPbased embedded system adopts unique dual-core structure and combines DSP core with high performance and low power dissipation with ARM microprocessor with strong control performance. So, it can offer necessary multimedia services in computer course teaching. On the other hand, a multimedia playing, and storage method is added in the embedded system, which not just greatly reduces storage space occupied by multimedia resources, but also offers multiple audio effect selections for users. Hence, it can better meet diversified and individualized needs so as to reach the better teaching effect.

\section{Theoretical construction}

OMAP-based embedded system is the foundation for development of various drive programs and application programs, including two major parts: mainframe software development environment and embedded Linux system software. It is widely applied in wireless communication, video conference and other fields [10]. According to startup feature of OMAP, the embedded system needs to complete mainframe development environment construction, UBL transplantation, UBoot transplantation and file system establishment. Moreover, a multimedia playing, and storage method is also introduced to reduce largely the storage resources occupied by multimedia, provide users with diversified dubbing options, satisfy individualized user needs and improve teaching efficiency as far as possible.

\subsection{Brief introduction to OMAP software mechanism}

Since multi-level BOOT is applied, the common startup way of OMAP is onboard NandFlash startup. The process is shown in in Fig.1: 


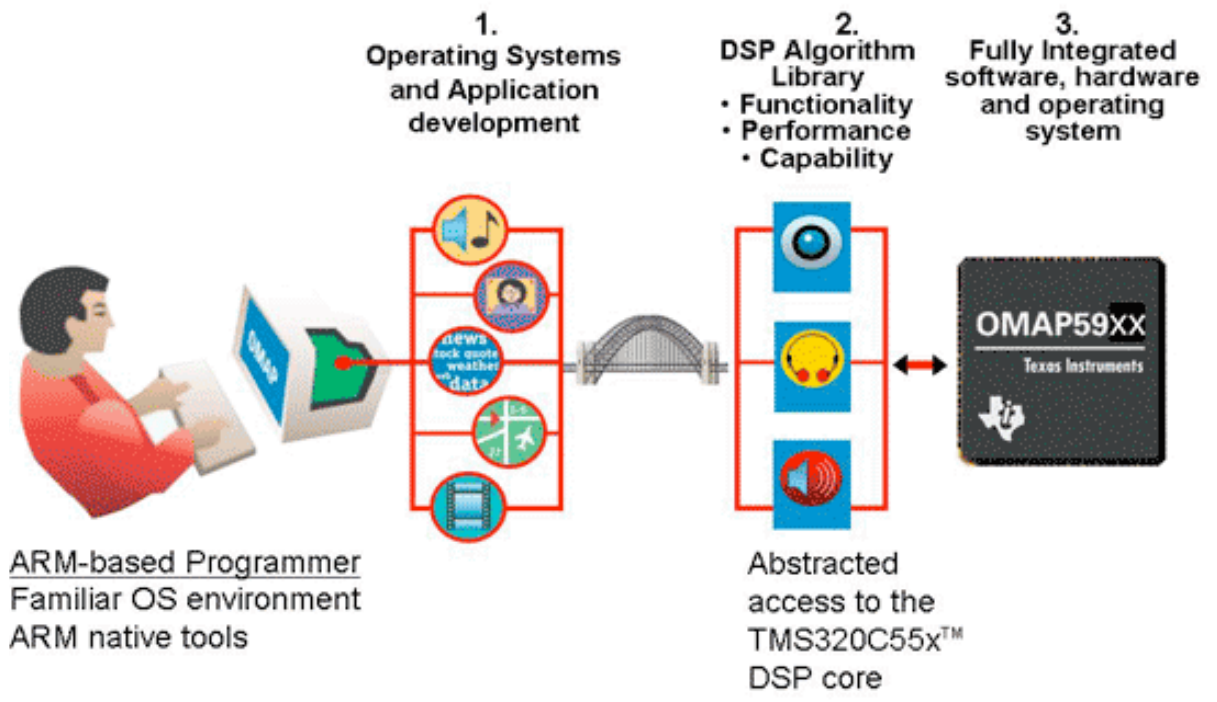

Fig. 1. Common startup way of OMAP

The procedures executed by system startup include [11]: DSP RBL, ARM RBL, UBL, UBoot, Linux Kernel and file system. Firstly, when starting up, OMAP defaults ARM core to be dormant, and DSP core to activate. So, DSP first starts up and reads ROM Bloader that only DSP core can access from DSP L2 ROM, i.e. DSP RBL. After DSP RBL makes some simple initialization, it will activate ARM core. Then, ARM will execute ARM RBL, load UBL program from Nand Flash's UBL initial address and operate UBL. Thirdly, UBL conducts some initialization work and skips to UBoot entrance to execute UBoot. Fourthly, UBoot reads in core startup data from Nand Flash and loads Linux. Finally, Linux core starts up, mounts the file system stored in Nand Flash and completes file system execution.

\subsection{Construction of mainframe development environment}

Mainframe development environment includes operating system, compilation tool and integrated development environment. Since OMAP is based on DSP and ARM dual-core processor, mainframe development environment also includes Windows and Linux. Windows system is responsible for operating file making tools, and hyperterminal debugging tools. Linux system mainly provides cross compiling tool, TFTP service and NFS service for system development. In this paper, the construction process of Linux system development environment is mainly introduced.

Firstly, install Linux operating system: install VirtualBox software and Ubuntu system;

Secondly, install OMAP development environment: with the help of original installation package; 
Thirdly, provide TFTP service: first, install TFTP software and then configure the server:

TFTP_USERNAME="tftp"

TFTP_DIRECTORY="/home/us er name/tftpboot"

TFTP_OPTIONS="-1-c-s"

Fourthly, provide NFS service: firstly install the software, then configure services and add the following at the end of corresponding file:

/home/username/workdir/filesys*(rw,sync,no_root_squash)

TFTP is a simple protocol to transmit small files. NFS is network file system and achieves network intercommunication through sharing a file system online. It is especially suitable for the need of embedded development.

\subsection{UBL and UBoot transplantation}

UBL program is stored in Nand Flash. It operates by loading ROM-cured ARM RBL program to the storage in OMAP. UBL program further adopts startup mode to load UBoot program to RAM from the specified site and finally operates on UBoot. UBoot is a general startup program. It is usually applied to initialize hardware equipment, establish memory mapping and prepare for loading and operating Linux core.

UBL transplantation includes four steps: modify, compile, convert out file into AIS file, and program UBL with nandwriter. During programming UBL with nandwriter, nandwriter file needs to be copied to windows first, and then NAND reused pin is set to program. UBoot transplantation includes the following steps: simplify, try to compile, modify, compile and program. After UBL transplantation and UBoot transplantation are completed, both have been programmed to NAND Flash and can play a role in the system.

\subsection{Establishment of file system}

Establishment of file system includes two steps: make and program. In the embedded Linux, due to the limit of storage capacity, file system must go through redesign and cut-down. In practical work, Busybox is often used to cut down file system, and then the file system is improved as needed. If it is required to implement independent operation of the system, relevant tools are needed to make the file system into mapping file and program it into Flash.

\subsection{Introduction of multimedia playing method, playing device and storage method}

Since the existing technology consumes numerous storage resources and cannot meet individualized appreciation needs, the special multimedia playing method is introduced, playing device and storage method are introduced to enhance the application effect of OMAP-based embedded system. The introduction is as follows:

Firstly, multimedia playing method. It includes the following steps: (1) acquire multimedia file information and dubbing configuration confirmed by users. File in- 
formation includes multimedia video, background audio, and storage information of dubbing text. Dubbing configuration includes vocal print features of each role; (2) acquire multimedia video, background audio, and dubbing text according to file information; (3) generate dubbing audio according to dubbing text and dubbing configuration, and match vocal print features of each role with vocal print features in dubbing configuration; (4) synthesize dubbing audio and background audio.

Secondly, multimedia playing device. The module structure is shown in the fig. 2 :

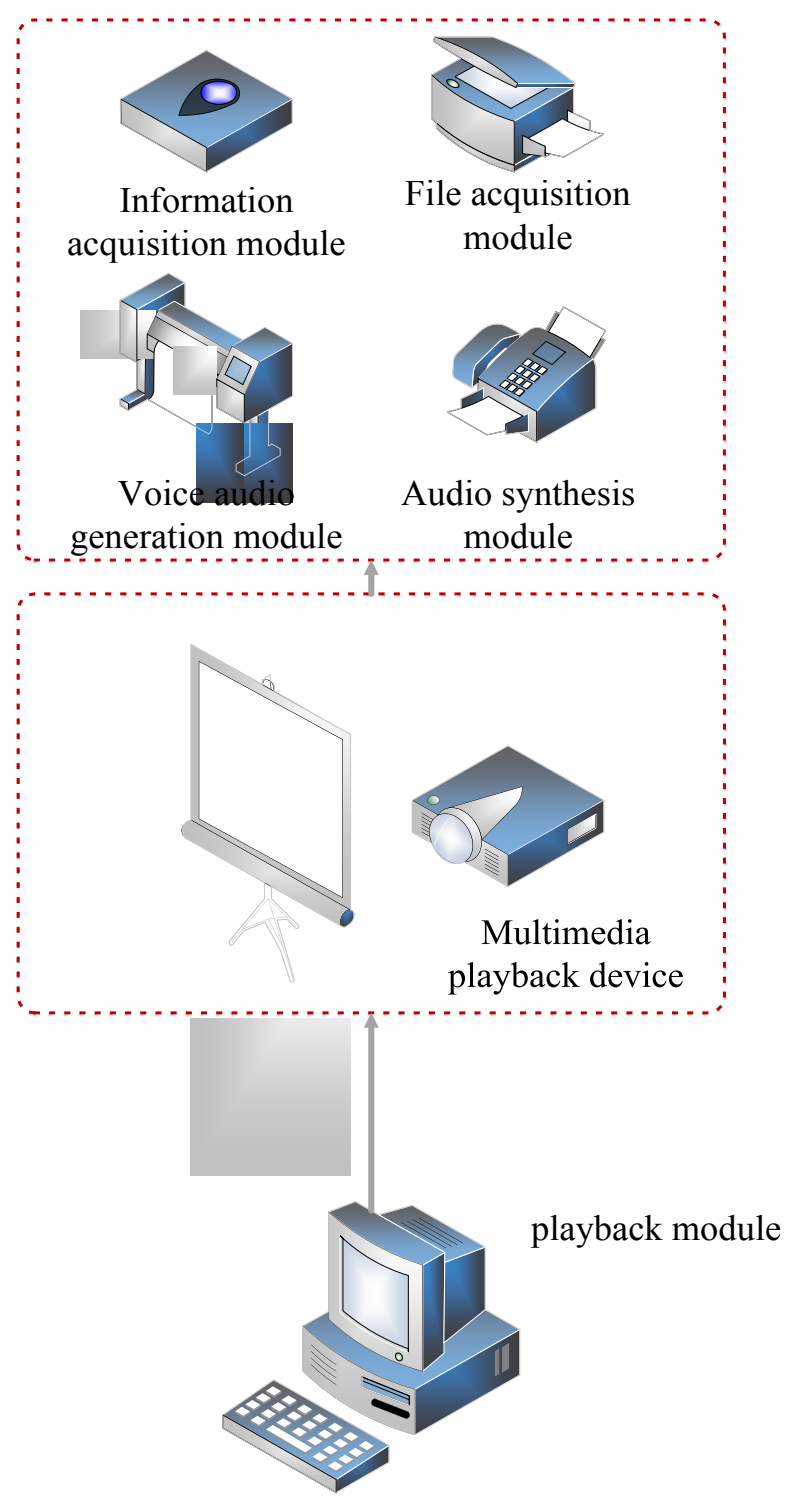

Fig. 2. Module structure of multimedia playing device 
Information acquisition module, file acquisition module, dubbing audio generation module and audio synthesis module correspond to multimedia playing method. The playing module is used to synchronously play multimedia video and audio. Besides, with system construction continuously goes deep, multiple languages and even dialects will be introduced in dubbing configuration.

Finally, multimedia storage method: extract video and audio in the original multimedia file, then divide background audio and dubbing audio, covert the divided dubbing audio into dubbing text, and store video, background audio and dubbing text respectively so as to largely save storage space. The diagram of multimedia storage method is as Fig. 3;

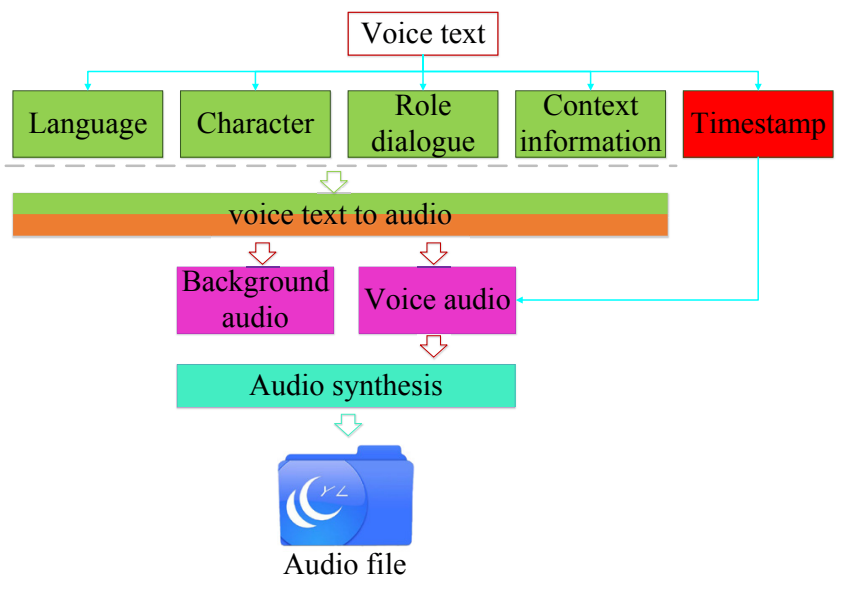

Fig. 3. Diagram of multimedia storage method

\section{Construction of Computer experiment teaching system based on OMAP embedded system}

\subsection{Function design for teacher terminal}

Function design for teacher terminal of OMAP embedded system is as follows:

Screen broadcast: teachers may send mainframe screen synchronously to students' mobile phone and conduct remote display of the teaching process. Students can watch the demonstration on their seat. Besides, in the whole operation process, teachers can choose electronic pointer and punctuate the key and difficult points for students.

Screen monitoring: teachers may watch students' main screen through monitoring function so as to pay attention to their learning dynamics in time, prevent them from losing their concentration and avoid the impact on teaching quality.

Assignment issuing: after completing experimental course teaching of each chapter, teachers send the assignments to student terminal through the issuing function. 
Force to quit: to avoid wrong login, the teacher terminal is also equipped with the function of forcing to quit. Teachers may make students to force to quit according to students' feedbacks.

Remote shutdown: after classroom teaching ends, teachers can control all equipment through remote shutdown. Teacher management module is shown in Fig.4.

It can be seen from Fig.4 that, (1) "teacher login" sub-module. The teacher inputs login username and password to send login request to the sever. After "information login certification module" confirms the login information is correct, the teacher may directly enter teacher teaching management homepage. (2) "Course data management" sub-module. The teacher may utilize "online course data check" to check teaching plans, course materials and experiment materials of the course, or make CAI courseware offline. Then, the teacher may upload CAI courseware to the virtual directory of aided teaching site through "data transmission unit". Uploading common documents such as PPT, DOC, PDF and HTML is supported. (3) "Online examination management" sub-module. The teacher may utilize "test base management unit" to manage test base and apply "automatic question extracting unit" to set question type, number and difficulty. The system automatically generates test paper at random and corresponding standard answers. (4) "Message management" sub-module. The teacher may utilize "message editing module" and "message module" to answer students" questions and manage the replied messages.

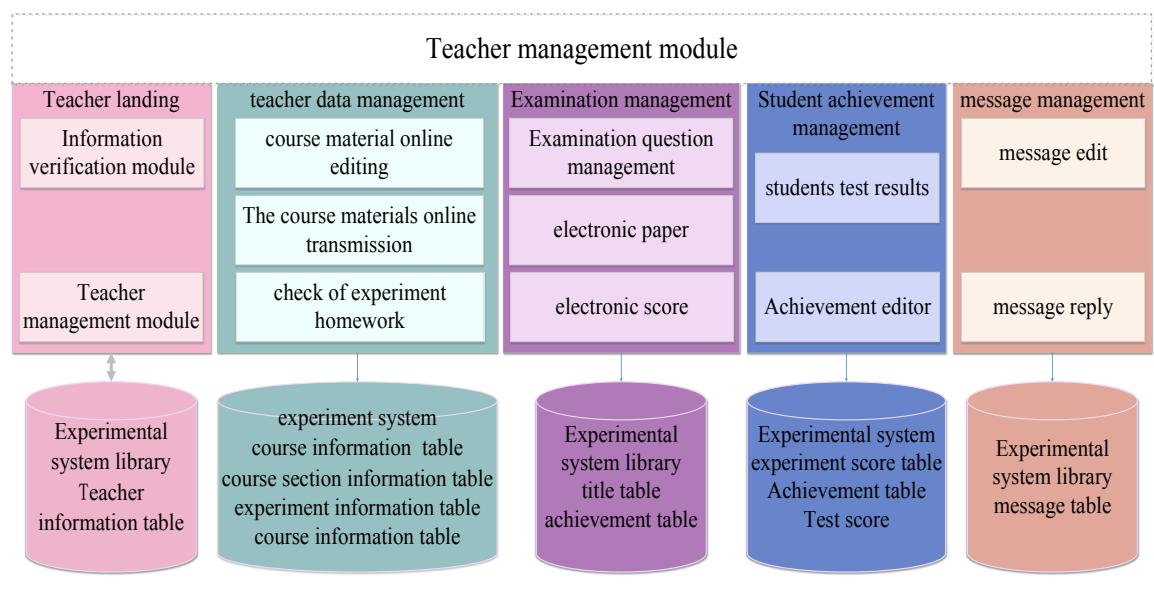

Fig. 4. Diagram of teacher management module

\subsection{Function design of student terminal}

Function design of student terminal in OMAP-based embedded system is as follows:

1. Electronic sign-in: students sign in through the tool bar at student terminal so that the teacher can check the attendance; 
2. Electronic questioning: students can ask questions through electronic hand raising to solve their bounds in the learning process.

3. Assignment submission: after students complete the assignment online, they may click assignment submission button to transmit the assignment to the teacher terminal.

4. Multimedia playing: students may choose multimedia file to demonstrate the experimental content again so as to enhance learning effect.

Function design of Computer experiment teaching system based on OMAP embedded system is shown in the Fig. 5 .

The login interface of Computer experiment teaching system is shown in Fig. 6.

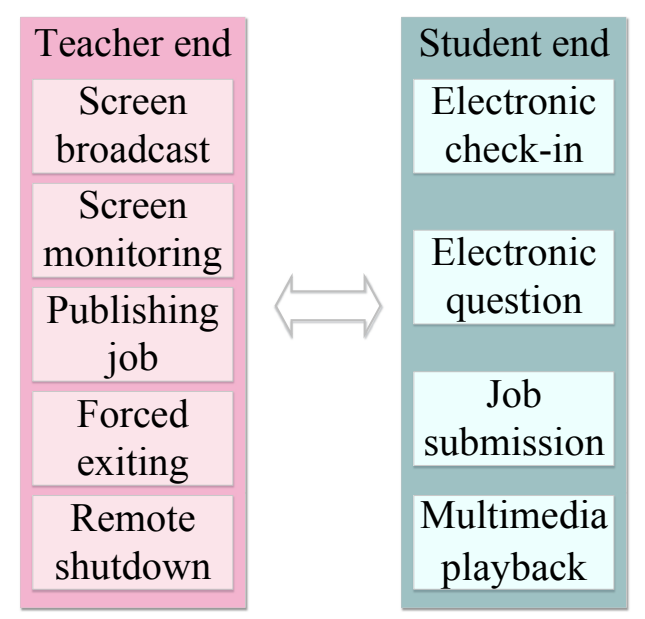

Fig. 5. Function design of OMAP embedded system

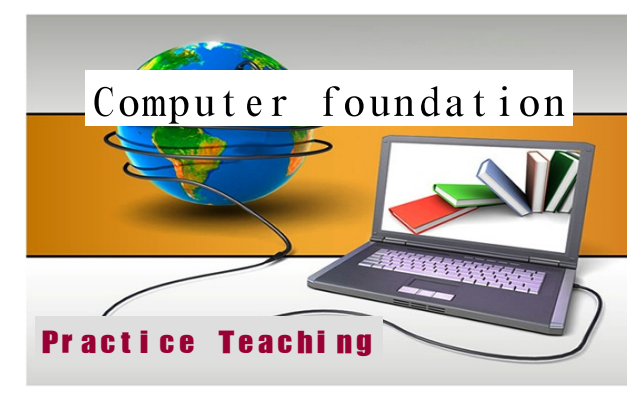

Fig. 6. Login interface of Computer experiment teaching system

\subsection{Effect check}

To verify the application effect of OMAP embedded system in practical teaching, the following experiment is designed. The sophomores in the school of computer of a university were taken as the object of study. 2 classes (42 students and 47 students) 
were extracted at random as the experimental class. Computer experiment teaching system based on OMAP embedded system was applied for the e experimental class. Meanwhile, the other 2 classes (47 students and 46 students) were extracted at random as the control class. Traditional teaching system was used for the control class. The teacher and teaching conditions of both classes had no significant difference. The age, gender, physical quality and comprehensive ability of students in both classes had no significant difference. The teaching scene of experimental course is as Fig. 7 and Fig. 8:

Basis of Computer Engineering was chosen as the experimental course. The teaching contents covered all kinds of computer software and hardware knowledge, including computer peripheral equipment, operating system, file management and office software. The teaching objective of Basis of Computer Engineering was to make students master essential basic knowledge of computer and basic skills, and own preliminary computer application ability.

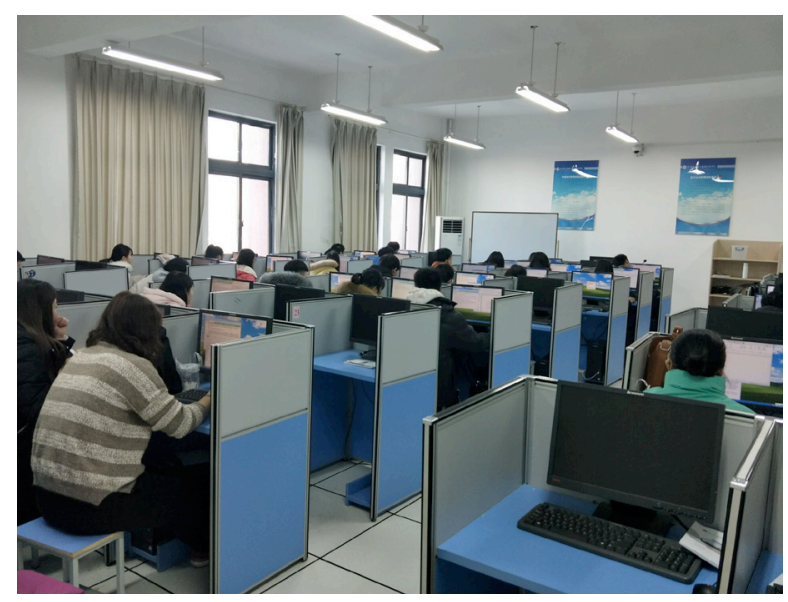

Fig. 7. Teaching scene of experiment course (1)

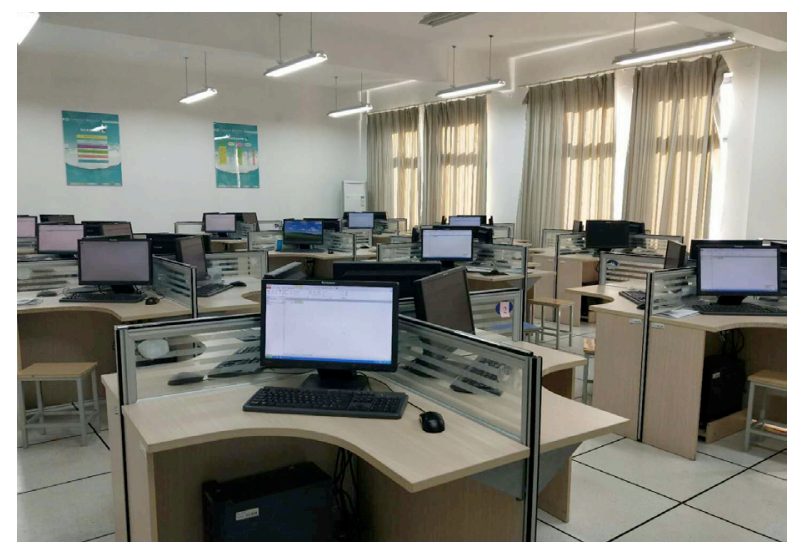

Fig. 8. Teaching scene of experiment course (2) 
After the course ended, special examination was conducted for students. Besides, comparative analysis was carried out for experimental class and control class. The test paper is set as Table 1 .

Teaching-examination separation mode was adopted for the assessment. The final examinational teachers were other teachers except the course teachers. The scoring was unrelated to course performance, so the assessment was more objective and fair. The scores were classified into four levels: excellent, good, medium and pass. The statistical results are as table 2 :

Table 1. Test paper setting

\begin{tabular}{|c|c|c|c|c|c|c|c|}
\hline Content & $\begin{array}{c}\text { Basic } \\
\text { knowledge }\end{array}$ & $\begin{array}{c}\text { Operating } \\
\text { system }\end{array}$ & $\begin{array}{c}\text { Internet } \\
\text { application }\end{array}$ & Word & Excel & PPT & $\begin{array}{c}\text { Multimedia soft- } \\
\text { ware application }\end{array}$ \\
\hline Percentage & $20 \%$ & $15 \%$ & $10 \%$ & $15 \%$ & $15 \%$ & $15 \%$ & $10 \%$ \\
\hline
\end{tabular}

Table 2. Statistical results of examination scores

\begin{tabular}{|l|c|c|c|c|}
\hline \multicolumn{1}{|c|}{ Class } & Excellent & Good & Medium & Pass \\
\hline Control class 1 $(\mathrm{n}=47)$ & $0(0 \%)$ & $11(23.40 \%)$ & $27(57.45 \%)$ & $9(19.15 \%)$ \\
\hline Control class 2 $(\mathrm{n}=46)$ & $0(0 \%)$ & $15(32.61 \%)$ & $20(43.48 \%)$ & $11(23.91 \%)$ \\
\hline Average Scores & $0(0 \%)$ & $13(28.01 \%)$ & $23.5(50.46 \%)$ & $10(21.53 \%)$ \\
\hline Experimental class 1 $(\mathrm{n}=42)$ & $3(7.14 \%)$ & $14(33.33 \%)$ & $22(52.38 \%)$ & $3(7.14 \%)$ \\
\hline Experimental class 2 $(\mathrm{n}=40)$ & $4(10 \%)$ & $21(52.50 \%)$ & $13(32.50 \%)$ & $2(5.00 \%)$ \\
\hline Average Scores & $3.5(8.57 \%)$ & $17.5(42.92 \%)$ & $17.5(42.44 \%)$ & $2.5(6.07 \%)$ \\
\hline
\end{tabular}

It is known from the table that, the score of experimental class is significantly higher than that of control class. The number of students gaining excellent result improves a lot. The mean excellence rate of experimental class achieves the breakthrough of zero, up to $8.57 \%$, and the good rate of experimental class is also $14.91 \%$ higher than that of control class. The number of students with low scores decreases a lot. The medium rate and pass rate decrease by $8.02 \%$ and $15.46 \%$ on the average. In addition, normal distribution of scores of 'experimental class' is more rational, and students generally had keen interest in the basic knowledge of computer. After course teaching was finished, some students in the experimental class took the initiative to conduct in-depth investigative study.

In general, OMAP-based embedded system conforms to teaching requirements of Basis of of Computer Engineering, and achieves the good teaching effect. In addition, it plays a great role in achieving the teaching reform objective and improving teaching quality. Therefore, the application prospect of OMAP-based embedded system and other multimedia commuter technology in computer course teaching, and it has great promotion value. 


\section{Conclusions}

College computer course teaching pays attention to experiment teaching, and puts forward the higher requirements for the application of multimedia computer technology. Hence, the development of OMAP-based embedded system has important significance for experiment teaching of computer courses. Based on practical needs of computer teaching, OMAP-based embedded system was designed and implemented from the perspectives of application and technology in this paper. From the perspective of performance, the whole system has reached the expected design objective, and can satisfy computer experiment teaching requirements. Meanwhile, the introduction of multimedia playing method, playing device and storage method can largely reduce storage resources occupied by multimedia and offer individualized multimedia service for users so as to reach the better application effect. Computer course teaching based on OMAP embedded system effectively cultivates students' interest in computer software and hardware knowledge and promotes their information accomplishment. Students can know computer-related information morality and information security and lay a good foundation for future work while mastering essential knowledge and skills.

\section{$6 \quad$ References}

[1] Luo, H.L., Shyu, M.L. Quality of service provision in mobile multimedia - a survey. Human-centric Computing and Information Sciences, 2011, vol. 1(1), pp. 5. https://doi.org/10.1186/2192-1962-1-5

[2] Ojala, T. Case studies on context-aware mobile multimedia services. Journal of Digital Information Management, 2010, vol. 8(1), pp. 4-15.

[3] Karande A M, Chunekar V N, Meshram B B. Working of Web Services using SOA[J]. International Journal of Advanced Research in Computer Science, 2010, 01(04).

[4] Yuan S T, Lu M R. An value-centric event driven model and architecture: A case study of adaptive complement of SOA for distributed care service delivery[J]. Expert Systems with Applications, 2009, 36(2):3671-3694. https://doi.org/10.1016/j.eswa.2008.02.024

[5] Nascimento A S, Rubira C M, Burrows R, et al. Designing fault-tolerant SOA based on design diversity[J]. Journal of Software Engineering Research \& Development, 2014, 2(1):13. https://doi.org/10.1186/s40411-014-0013-7

[6] Liang L I, Qiong L U. Design of Distributed Video Retrieval System Based on SOA Technology[J]. Video Engineering, 2014.

[7] Toda K. Skill development for SOA,MDA and Trial for Software Production Engineering[J]. Ieice Technical Report, 2008, 107:7-12.

[8] Reyes-Delgado P Y, Mora M, Duran-Limon H A, et al. The strengths and weaknesses of software architecture design in the RUP, MSF, MBASE and RUP-SOA methodologies[J]. Computer Standards \& Interfaces, 2016, 47(C):24-41. https://doi.org/10.1016/j.csi.2016.02 .005

[9] Zhao H Q, Sun J. A Methodological Study of Evaluating the Dependability of SOA Software System[J]. Chinese Journal of Computers, 2010, 33(11):2202-2210. https://doi.org/10.3724/SP.J.1016.2010.02202 
[10] Paulsson F, Berglund M, Arora A S. Suggesting an SOA Framework for Modular Virtual Learning Environments: Comparing Two Implementation Approaches[J]. International Journal of Web-Based Learning and Teaching Technologies (IJWLTT), 2008, 3:43-57. https://doi.org/10.4018/jwltt.2008010105

[11] Paik H Y, Rabhi F A, Benatallah B, et al. Service Learning and Teaching Foundry: A Virtual SOA/BPM Learning and Teaching Community[J]. 2010, 66:790-805.

[12] Tere G M, Jadhav B T. Design Patterns For Successful Service Oriented Architecture Implementation[J]. Bvicams International Journal of Information Technology, 2010, 2(2).

[13] Domdouzis K, Andrews S, Akhgar B, et al. Application of a New Service-Oriented Architecture (SOA) Paradigm on the Design of a Crisis Management Distributed System[J]. International Journal of Distributed Systems \& Technologies, 2016, 7(2):1-17. https://doi.org/10.4018/IJDST.2016040101

\section{$7 \quad$ Authors}

Xiaorong Wang is an Associate Professor in Jiangsu College of Finance and Accounting, Lianyungang 223003, China.

Cuimei Fan is a lecturer in Jiangsu College of Finance and Accounting, Lianyungang 223003, China.

Article submitted 13 February 2018. Resubmitted 18 March 2018. Final acceptance 27 March 2018. Final version published as submitted by the authors. 